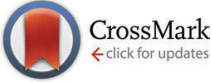

Cite this: Phys. Chem. Chem. Phys., $2015,17,23554$

Received 2nd July 2015, Accepted 18th August 2015 DOI: $10.1039 / c 5 c p 03821 a$

www.rsc.org/pccp

\title{
Adsorption of 2-propanol on ice probed by ambient pressure X-ray photoelectron spectroscopy $\dagger$
}

\author{
John T. Newberg ${ }^{a}$ and Hendrik Bluhm* ${ }^{\text {b }}$
}

\begin{abstract}
The interaction of 2-propanol with ice was examined via ambient pressure X-ray photoelectron spectroscopy (APXPS), a surface sensitive technique that probes the adsorbed 2-propanol directly with submonolayer resolution. Isothermal uptake experiments were performed on vapor deposited ice at $227 \mathrm{~K}$ in the presence of the equilibrium water vapor pressure of 0.05 Torr and 2-propanol partial pressures ranging from $5 \times 10^{-5}$ to $2 \times 10^{-3}$ Torr. The C 1 s APXPS spectra of adsorbed 2-propanol showed two characteristic peaks associated with the $\mathrm{C}_{\mathrm{OH}}$ alcohol group and $\mathrm{C}_{\mathrm{Me}}$ methyl groups in a 1:2 ratio, respectively. Coverage increased with 2-propanol partial pressure and followed first order Langmuir kinetics with a Langmuir constant of $K=6.3 \times 10^{3}$ Torr $^{-1}$. The $1: 2$ ratio of $\mathrm{C}_{\mathrm{OH}}: \mathrm{C}_{\mathrm{Me}}$ remained constant with increasing coverage, indicating there is no chemical reaction upon adsorption. The observed Langmuir kinetics using APXPS is consistent with previous observations of other small chain alcohols via indirect adsorption methods using, e.g., Knudsen cell and coated wall flow tube reactors.
\end{abstract}

\section{Introduction}

The processing of volatile organic carbon (VOC) compounds within environmental ices produces humic-like substances and can significantly impact the surrounding environment by affecting the oxidizing capacity of the atmosphere and contributing to secondary aerosol formation. ${ }^{1}$ There is increasing knowledge regarding the interactions of inorganic and organic vapors with ice surfaces. ${ }^{1-6}$ However, most of the experimental adsorption studies to date probe adsorption at the ice surface indirectly by monitoring the decrease of the partial pressure of the adsorbing species in the gas phase. In the present study we use ambient pressure X-ray photoelectron spectroscopy (APXPS) to probe the surface directly under steady-state adsorption-desorption conditions. APXPS is a surface sensitive tool that is chemically specific and probes the top few nanometers of a surface with submonolayer coverage sensitivity. APXPS has been used previously to investigate the interface of vapor deposited ice, ${ }^{7} \mathrm{NaCl}$ water/ice, ${ }^{8}$ and ice in the presence of gas phase acetic acid, ${ }^{9}$ acetone, ${ }^{10}$ and $\mathrm{NO}_{2} \cdot{ }^{11}$

Previous alcohol adsorption studies on ice were performed using, e.g., a Knudsen cell, ${ }^{12}$ coated wall flow tube $(\mathrm{CWFT})^{13-18}$ and Brunauer-Emmett-Teller (BET) adsorption methods. ${ }^{14}$ The alcohols investigated include methanol, ${ }^{12,18}$ ethanol, ${ }^{13-17}$

\footnotetext{
${ }^{a}$ University of Delaware, Department of Chemistry and Biochemistry, Newark, DE 19716, USA.E-mail: jnewberg@udel.edu

${ }^{b}$ Chemical Sciences Division, Lawrence Berkeley National Laboratory, Berkeley, CA 94720, USA. E-mail: hbluhm@lbl.gov

$\dagger$ Electronic supplementary information (ESI) available. See DOI: 10.1039/c5cp03821a
}

1-propanol, ${ }^{16}$ 1-butanol, ${ }^{16}$ 1-pentanol, ${ }^{16}$ and 1-hexanol ${ }^{16}$ at temperatures $>150 \mathrm{~K}$ with alcohol partial pressures ranging from about $5 \times 10^{-7}$ to $5 \times 10^{-4}$ Torr. Submonolayer coverages of C1 to C4 alcohols were observed under these conditions with first order Langmuirian kinetics. ${ }^{12-18}$ For comparison, typical urban levels of $\mathrm{C} 1$ to $\mathrm{C} 3$ alcohols range from approximately 1 to $1400 \mathrm{ppb}^{19,20}$ (i.e., $\sim 10^{-6}$ to $10^{-3}$ Torr partial pressures). Thus, the aforementioned ice adsorption studies were performed in pressure regimes indicative of "dirty" ice, ${ }^{21}$ conditions typical for ice surfaces exposed to high levels of anthropogenic emissions in urban environments.

In this study we investigate the isothermal uptake of 2-propanol on ice at $227 \mathrm{~K}$ with partial pressures from $5 \times 10^{-5}$ to $2 \times 10^{-3}$ Torr using APXPS. While the adsorption of a number of different alcohols on ice has been investigated under similar conditions, ${ }^{12-18}$ to our knowledge this is the first study of 2-propanol adsorption on ice. Experiments were performed at $227 \mathrm{~K}$ in order to compare the results of this study to previous work on different alcohols at the same temperature. Alcohol adsorption on ice is studied here by probing the adsorbed species at the ice surface directly via APXPS, thus allowing for the determination of possible reactions of the adsorbed alcohol with the ice substrate.

\section{Experimental}

The experiments were performed using the APXPS endstation ${ }^{22}$ at the Molecular Environmental Science beamline (11.0.2) ${ }^{23}$ at the Advanced Light Source in Berkeley, CA. Ice samples with a 
thickness of $\sim 0.5 \mathrm{~mm}$ were grown through condensation of water vapor onto a $3 \mathrm{~mm}$ diameter $\mathrm{Cu}$ substrate, which was temperature controlled at $227 \mathrm{~K}$ using a custom-built Peltier sample holder with an integrated K-type thermocouple. ${ }^{22}$ Water vapor was admitted to the APXPS measurement chamber through a precision leak valve. The water source was the vapor above liquid water (Alfa Aesar, HPLC grade) in a vacuum sealed glass bulb. For each experiment the water supply was purified with at least three freeze-pump-thaw cycles. 2-Propanol vapor was admitted through a second precision leak valve using the vapor pressure above liquid 2-propanol (Sigma-Aldrich, >99.9\%) in a vacuum sealed glass bulb which was also freeze-pump-thawed three times prior to experiments. The total (water +2 -propanol) pressure in the measurement chamber was monitored using an MKS capacitance manometer pressure gauge (precision 0.01 Torr). For pure ice films the water vapor pressure in the chamber corresponded to the literature value $e^{24}$ for the equilibrium water vapor pressure above ice at $227 \mathrm{~K}$ (0.05 Torr), confirming the correctness of the pressure and temperature measurements. The partial pressure of 2-propanol was measured using a quadrupole mass spectrometer (QMS; Stanford Research Systems, RGA 200) which was attached to the preparation chamber of the APXPS system by monitoring $m / z=45$. The measurement chamber and preparation chamber were connected through a variable leak valve. The calibration curve of the $m / z=45$ (m45) signal (in arbitrary units) versus the partial pressure of 2-propanol in the analysis chamber is shown in the ESI $\dagger$ (Fig. S1). The downstream m45 QMS partial pressure signal is proportional to the square of the upstream APXPS chamber pressure, caused by the conductance of the gas flow through the leak valve restriction. ${ }^{25}$

C 1s APXPS data were collected at an incident photon energy of $490 \mathrm{eV}$. The combined analyzer and beamline resolution was better than $0.2 \mathrm{eV}$. The incident photon flux density was kept to below $3 \times 10^{11}$ photons per $\mathrm{mm}^{2}$ to reduce the effect of beam damage, which manifests itself in the appearance of a peak at the low BE side in the $\mathrm{C}$ 1s spectra. ${ }^{10}$ For the experiments in this study, no significant beam-induced damage was observed.

\section{Results and discussion}

APXPS C 1s spectra for gas phase 2-propanol at room temperature and 2-propanol adsorbed on ice at $227 \mathrm{~K}$ are shown in Fig. 1. For ease of comparison of the gas phase and adsorbed spectrum the binding energy axis is aligned to zero for the peak due to the methyl groups $\left(\mathrm{C}_{1}\right.$ and $\mathrm{C}_{3}$ in Fig. 1 inset). The central carbon atom $\left(\mathrm{C}_{2}\right)$ for both the gas phase and adsorbed phase is shifted by $1.5 \mathrm{eV}$ to a higher binding energy, a characteristic shift for an alcohol group. ${ }^{26}$ The methyl-to-alcohol C 1s intensity ratios are $2: 1$, as expected from the stoichiometry of the molecule. For acetone adsorption on ice ${ }^{10}$ the binding energy difference between the central carbonyl group and the terminating methyl groups (akin to $\mathrm{C}_{2}$ and $\mathrm{C}_{1,3}$ in the present case) was found to be $0.3 \mathrm{eV}$ higher for the adsorbed phase compared to the gas phase. These results are in contrast to what is observed for 2-propanol, where both the gas and adsorbed

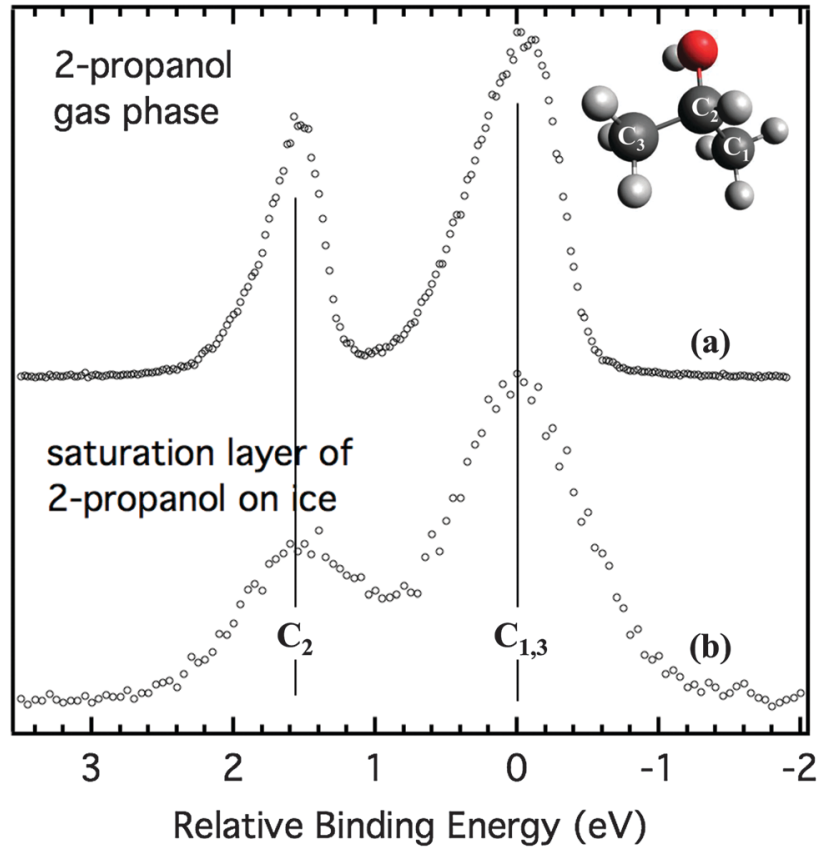

Fig. 1 C 1s spectra of (a) gas phase 2-propanol at 0.018 Torr and (b) adsorbed 2-propanol on ice at $227 \mathrm{~K}$ and 2-propanol partial pressure of 0.6 mTorr

phase have a similar binding energy difference of $1.5 \mathrm{eV}$ between $\mathrm{C}_{2}$ and $\mathrm{C}_{1,3}$. The asymmetric broadening of the gas phase species can be attributed to the excitation of the $\mathrm{C}-\mathrm{H}$ and $\mathrm{C}-\mathrm{O}$ stretching modes by the emitted photoelectrons. ${ }^{10}$ In going from the gas phase to the adsorbed phase, the $\mathrm{C}$ 1s peaks additionally broaden. This broadening could be due to electronic interactions between the ice surface and carbon species, which would include an averaging over many different adsorption geometries with slightly different core level binding energies. However, there could also be broadening due to inhomogeneous charging at the interface of the ice surface. The extent to which each of these mechanisms contribute to broadening is difficult to assess.

Two isothermal uptake experiments on ice were performed at $227 \mathrm{~K}$ with 2-propanol partial pressures ranging from $5 \times 10^{-5}$ to $3 \times 10^{-3}$ Torr. Fig. 2 shows representative spectra as a function of increasing partial pressure. The binding energies are scaled to the bulk ice $1 b_{1}$ peak at $6.5 \mathrm{eV} .^{27}$ The overall $\mathrm{C} 1 \mathrm{~s}$ signal increases with increasing 2-propanol partial pressure due to 2-propanol adsorption. The $\mathrm{C}_{\mathrm{OH}}: \mathrm{C}_{\mathrm{Me}}$ alcohol-to-methyl ratio remained at $1: 2$ with increasing coverage, indicating that 2-propanol adsorbs intact and does not strongly react with the ice surface.

A quantitative assessment of the amount of adsorbed 2-propanol as a function of partial pressure is shown in Fig. 3 for two independent uptake experiments, plotting the integrated $\mathrm{C} 1 \mathrm{~s}$ intensity versus 2-propanol partial pressure. The non-zero C $1 \mathrm{~s}$ intensity at the lowest 2-propanol pressures is due to the lack of a measurable m45 signal at the beginning of the adsorption experiment, below about $5 \times 10^{-5}$ Torr partial pressure in the analysis chamber. The difference in the measured peak area as a function of pressure in the two isotherm experiments is attributed to a small variation in the sample-to-aperture 


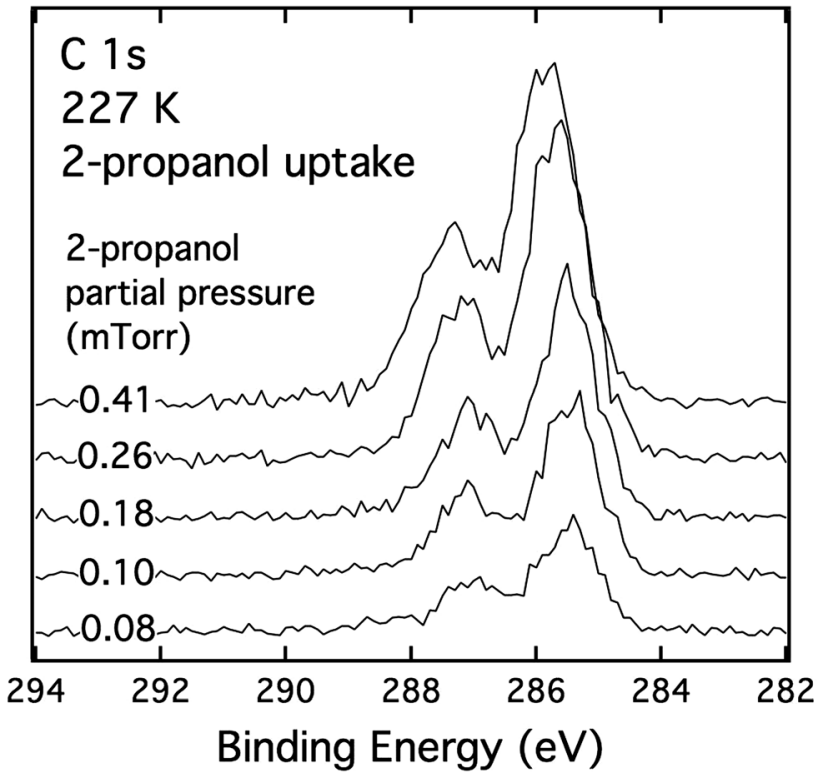

Fig. 2 C 1s APXPS spectra with increasing 2-propanol adsorption on ice at $227 \mathrm{~K}$.
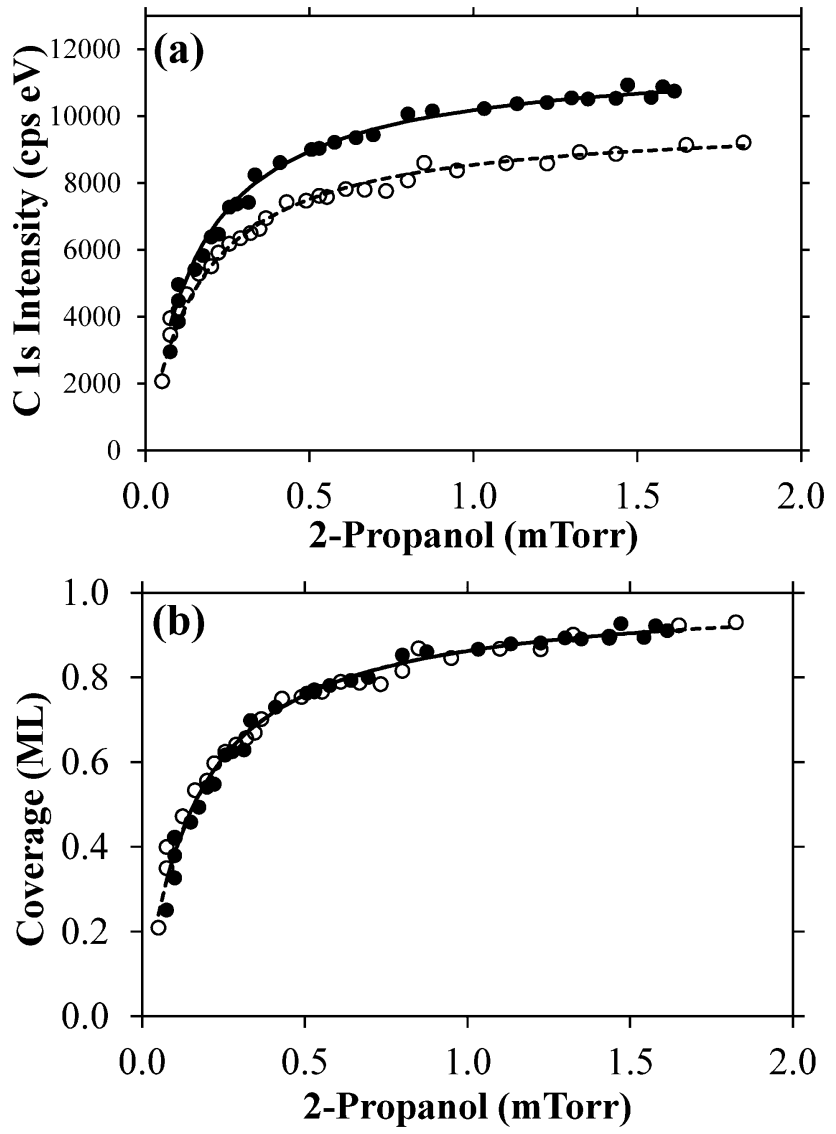

Fig. 3 Langmuir isotherms plotted as the (a) integrated $C$ 1s 2-porpanol intensity and (b) 2-propanol coverage as a function of vapor pressure at $227 \mathrm{~K}$. Solid and open symbols represent two independent experiments. Solid and dashed lines are first order Langmuir fits through the data.

distance and thus electron scattering by the gas phase from one uptake experiment to the next.
The smooth monotonic increase in 2-propanol intensity is indicative of Langmuirian adsorption-desorption kinetics observed previously with other alcohols adsorbed on ice. ${ }^{12-18}$ Multilayer uptake would lead to an inflection point in the uptake curve. ${ }^{16}$ Thus, the coverages are expected to be submonolayer approaching surface saturation. The lines fit through the data in Fig. 3a are first order Langmuir curves given by:

$$
I=I_{\mathrm{sat}} \frac{\mathrm{p} K}{1+\mathrm{p} K}
$$

where $I$ is the intensity from the integrated areas of C 1s peaks, $I_{\text {sat }}$ is the saturation intensity, $\mathrm{p}$ is the 2-propanol pressure and $K$ is the Langmuir constant, which is the ratio of the adsorption rate constant over the desorption rate constant. Thus, the larger the $K$, the greater the partitioning of the gas molecule to the surface. For submonolayer coverages $I \propto \sigma$, where $\sigma$ is the surface density of 2-propanol on the ice surface. Eqn (1) can then be rewritten as:

$$
\Theta=\frac{\sigma}{\sigma_{\text {sat }}}=\frac{\mathrm{p} K}{1+\mathrm{p} K}
$$

where $\Theta$ is the coverage of 2-propanol. Fig. 3b plots the coverage as a function of 2-propanol partial pressure. As seen from the results, the two independent uptake curves give rise to the same Langmuirian behavior with a value of $K=6.3 \times 10^{3} \mathrm{Torr}^{-1}$.

Fig. 4 compares the uptake of 2-propanol measured here using APXPS to CWFT studies of ethanol, 1-propanol and 1-butanol. ${ }^{16}$ To our knowledge, the paper by Sokolov and Abbatt is the only study to date that considers $\mathrm{C}_{3}$ and $\mathrm{C}_{4}$ alcohol adsorption on ice and is thus a relevant reference for the present investigation to compare to. The uptake data in the CWFT studies were reported as $\sigma$ (molecules $\mathrm{cm}^{-2}$ ) versus $p$ (Torr). For Fig. 4 the CWFT data (open circles) were extracted from ref. 16 using WebPlotDigitizer Version 3.8 in the reported units and then converted to coverage (ML) by dividing by the reported $\sigma_{\text {sat }}$ values for each alcohol. The Langmuir fits to the data (solid lines) were generated using eqn (2) with the reported values of $K=1.22 \times 10^{3}, 3.50 \times 10^{3}$ and $13.4 \times 10^{3} \mathrm{Torr}^{-1}$ for ethanol, 1-propanol and 1-butanol, respectively.

As seen from Fig. 4a (linear scale $x$-axis) APXPS experiments are able to probe roughly a factor of four higher alcohol partial pressures than CWFT experiments. The upper pressure limit in the CWFT experiments is determined by the potential for condensation onto the injector which is at a lower temperature than the ice surface. ${ }^{16}$ Thus, in an CWFT experiment the alcohol partial pressure must be kept significantly below the alcohol condensation point occurring at the cold injector temperature. For APXPS experiments the coldest point in the chamber is the Peltier element-cooled copper substrate which is covered by ice during the experiments. ${ }^{22}$ The APXPS 2-propanol experiments could have probed at high pressures, but were stopped near $\sim 0.002$ Torr because the $\mathrm{C} 1 \mathrm{~s}$ spectral intensity did not change much above this partial pressure. The ability to probe at high partial pressures allow for APXPS experiments to collect data near the adsorption saturation point, thus enabling a confident determination of $\sigma_{\text {sat }}$ from the first order Langmuir fit. 


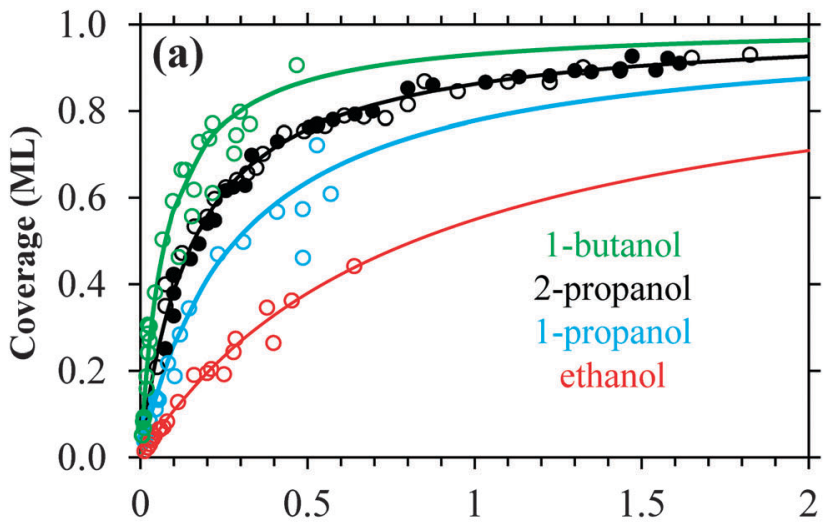

Alcohol Pressure (mTorr)

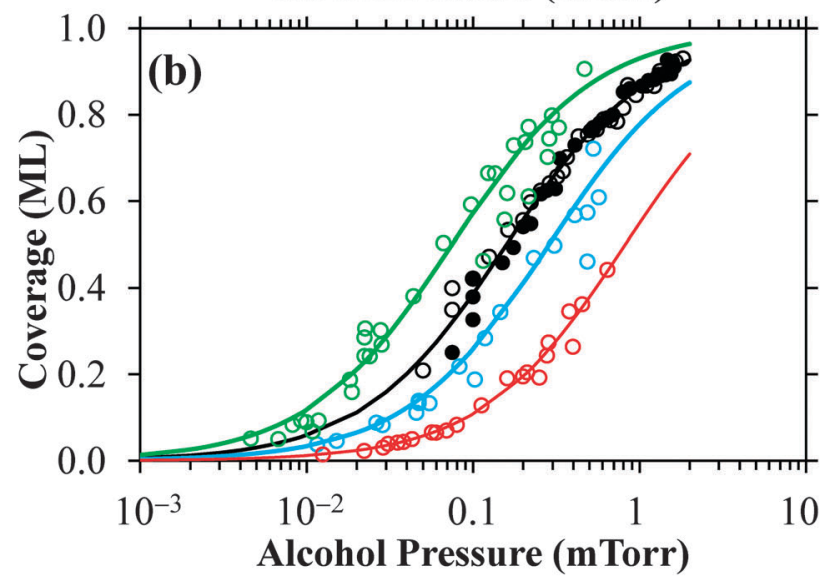

Fig. 4 Alcohol coverage as a function of partial pressure using a (a) linear $x$-axis and (b) $\log x$-axis. 2-Propanol data is from Fig. 3. 1-Butanol, 1-propanol and ethanol data are from ref. 16. Lines are first order Langmuir fits through the data.

Fig. 4b (log scale $x$-axis) shows that CWFT studies were able to probe roughly a factor of five to ten (depending on the alcohol) lower partial pressures than APXPS at a similar temperature. It should be noted that the lower pressure limit in the APXPS studies was not due to lack of C 1s signal but instead by QMS sensitivity; for example, the bottom spectrum in Fig. 2 was taken at $8 \times 10^{-5}$ Torr, where there is still a significant $\mathrm{C} 1 \mathrm{~s}$ intensity (signal-to-noise ratio of about ten), corresponding to a coverage of $\sim 0.2 \mathrm{ML}$.

The partial pressure detection limits for CWFT studies were reported to be in the range of $10^{-7}$ to $10^{-6}$ Torr, depending on the alcohol. ${ }^{16}$ The details of the CWFT setup ${ }^{28}$ show that the flow cell (where adsorption occurs) is separated from a QMS via two apertures and one differentially pumped stage. This allows for a direct line-of-sight from the flow cell into the QMS for detection. Assuming a detection limit near $10^{-10}$ Torr for the QMS and comparing this to the $10^{-5}$ Torr detection limit for 1-propanol (Fig. 4b, blue data), this gives a pressure differential of $10^{5}$ in going from the flow cell to the mass spectrometer, mainly due to the differentially pumped stage between the two. By comparison, there is roughly a factor of five greater loss $\left(\sim 5 \times 10^{5}\right)$ for 2-propanol detection in going from the analysis chamber in APXPS studies with a detection limit of $\sim 5 \times 10^{-5}$ Torr, assuming as well a detection limit of $10^{-10}$ Torr at the QMS. This fivefold greater loss in alcohol detection in APXPS studies (compared to $\mathrm{CWFT}^{16}$ ) can be overcome by improvements of the conductance from the analysis chamber to the QMS in future APXPS measurements.

The observed value of $K=6.3 \times 10^{3} \mathrm{Torr}^{-1}$ at $227 \mathrm{~K}$ for 2-propanol suggests that the partitioning of 2-propanol is greater than that of 1-propanol $\left(3.50 \times 10^{3} \mathrm{Torr}^{-1}\right)$, but less than that of 1-butanol $\left(13.4 \times 10^{3} \mathrm{Torr}^{-1}\right)$ at $227 \mathrm{~K}$. More specifically, comparing the two isomers 1- and 2-propanol, the latter appears to bind more strongly to ice. However, given that these observations are based on measurements using two different experimental techniques one should be cautious to draw any strong conclusions from this comparison. For example, APXPS experiments prepare ice surfaces through vapor deposition, while the CWFT studies in Fig. 4 freeze liquid water to generate a thin film of ice, so the influence of possible differences in the surface morphology on the observed adsorption behavior cannot be excluded.

Input from theoretical calculations will help to elucidate the reason behind the different adsorption energetics of the four molecules compared in Fig. 4. Ethanol adsorption on ice was examined using molecular dynamic (MD) simulations over the temperature range from $193 \mathrm{~K}$ to $223 \mathrm{~K}$ using the TIP4P water model. ${ }^{17}$ At submonolayer coverages, lateral H-bonding and alkylalkyl interactions were found to be minor. The major contribution to the total energy is ethanol-ice hydrogen bonding. At monolayer coverage and beyond lateral interactions become significant. Similar observations of strong ice- $\mathrm{OH}$ interactions for methanol at submonolayer coverages were found using MD (TIP4P) from $210 \mathrm{~K}$ to $250 \mathrm{~K}^{29}$ and Monte Carlo simulations at $200 \mathrm{~K}^{30}$ Thus, molecular simulations indicate that the ice-OH interaction dominates the overall adsorption energetics under submonolayer conditions, consistent with a Langmuirian mechanism where lateral interactions are negligible. Computer simulations comparing the adsorption energetics of 1-propanol, 2-propanol and 1-butanol could shed light on whether the observed difference in adsorption behavior is consistent with the APXPS and CWFT observations in Fig. 4.

\section{Conclusions}

2-Propanol adsorption on ice was examined using ambient pressure X-ray photoelectron spectroscopy (APXPS) at an ice temperature of $227 \mathrm{~K}$ and alcohol partial pressures ranging from $5 \times 10^{-5}$ to $2 \times 10^{-3}$ Torr. Previously, alcohol adsorption on ice was examined using coated wall flow tube, Knudsen cell and BET adsorption techniques which probe adsorption indirectly by measuring the loss from the gas phase. APXPS offers an advantage over these techniques by probing the surface chemistry directly with submonolayer resolution. APXPS C 1s spectra of adsorbed 2-propanol show two distinct peaks with an appropriate $1: 2$ stoichiometry for the alcohol group and two methyl groups. The coverage of 2-propanol increased with partial pressure above the ice surface. The adsorption mechanism followed first order Langmuir kinetics, which is consistent with negligible 
lateral interactions between adsorbed 2-propanol molecules and strong hydrogen bonding of the alcohol $\mathrm{OH}$ group to the ice surface. The observed Langmuir constant of $K_{\mathrm{p}}=6.3 \times$ $10^{3}$ Torr $^{-1}$ at $227 \mathrm{~K}$ suggests that the partitioning of 2-propanol to the ice surface is greater than 1-propanol but less than 1-butanol when compared to previous coated wall flow tube studies. ${ }^{16}$ However, given the use of two different experimental techniques to determine this difference in adsorption partitioning, additional studies and complementary molecular simulations are recommended to confirm these results.

\section{Acknowledgements}

This work was supported by the Office of Science, Biological and Environmental Research, Environmental Remediation Sciences Division (ERSD), U.S. Department of Energy under Contract No. DE-AC02-05CH11231. The ALS and the ALS-MES beamline 11.0.2 are supported by the Director, Office of Science, Office of Basic Energy Sciences, Division of Chemical Sciences, Geosciences of the U.S. Department of Energy at the Lawrence Berkeley National Laboratory under Contract No. DE-AC02-05CH11231. J.T.N. acknowledges support from NSF ANT-1019347.

\section{Notes and references}

1 V. F. McNeill, A. M. Grannas, J. P. D. Abbatt, M. Ammann, P. Ariya, T. Bartels-Rausch, F. Domine, D. J. Donaldson, M. I. Guzman, D. Heger, T. F. Kahan, P. Klan, S. Masclin, C. Toubin and D. Voisin, Atmos. Chem. Phys., 2012, 12, 9653. 2 C. Girardet and C. Toubin, Surf. Sci. Rep., 2001, 44, 163.

3 J. P. Abbatt, Chem. Rev., 2003, 103, 4783.

4 T. Huthwelker, M. Ammann and T. Peter, Chem. Rev., 2006, 106, 1375.

5 J. N. Crowley, M. Ammann, R. A. Cox, R. G. Hynes, M. E. Jenkin, A. Mellouki, M. J. Rossi, J. Troe and T. J. Wallington, Atmos. Chem. Phys., 2010, 10, 9059.

6 T. Bartels-Rausch, H. Jacobi, T. Kahan, J. L. Thomas, E. Thomson, J. Abbatt, M. Ammann, J. Blackford, H. Bluhm and C. Boxe, Atmos. Chem. Phys., 2014, 14, 1587.

7 H. Bluhm, D. Ogletree, C. Fadley, Z. Hussain and N. Salmeron, J. Phys.: Condens. Matter, 2002, 14, L227.

8 A. Krepelova, T. Huthwelker, H. Bluhm and M. Ammann, ChemPhysChem, 2010, 11, 3859.

9 A. Krepelova, T. Bartels-Rausch, M. A. Brown, H. Bluhm and M. Ammann, J. Phys. Chem. A, 2013, 117, 401.
10 D. E. Starr, D. Pan, J. T. Newberg, M. Ammann, E. G. Wang, A. Michaelides and H. Bluhm, Phys. Chem. Chem. Phys., 2011, 13, 19988.

11 A. Krepelova, J. T. Newberg, T. Huthwelker, H. Bluhm and M. Ammann, Phys. Chem. Chem. Phys., 2010, 12, 8870.

12 P. K. Hudson, M. A. Zondlo and M. A. Tolbert, J. Phys. Chem. A, 2002, 106, 2882.

13 A. Symington, L. M. Leow, P. T. Griffiths and R. A. Cox, J. Phys. Chem. A, 2012, 116, 5990.

14 J. P. D. Abbatt, T. Bartels-Rausch, M. Ullerstam and T. J. Ye, Environ. Res. Lett., 2008, 3, 045008.

15 M. Kerbrat, S. Le Calve and P. Mirabel, J. Phys. Chem. A, 2007, 111, 925.

16 O. Sokolov and J. Abbatt, J. Phys. Chem. A, 2002, 106, 775.

17 N. Peybernes, S. Le Calve, P. Mirabel, S. Picaud and P. N. M. Hoang, J. Phys. Chem. B, 2004, 108, 17425.

18 A. K. Winkler, N. S. Holmes and J. N. Crowley, Phys. Chem. Chem. Phys., 2002, 4, 5270.

19 G. Legreid, J. B. Lööv, J. Staehelin, C. Hueglin, M. Hill, B. Buchmann, A. S. Prevot and S. Reimann, Atmos. Environ., 2007, 41, 8409.

20 H. T. Nguyen, N. Takenaka, H. Bandow, Y. Maeda, S. T. de Oliva, M. M. Botelho and T. M. Tavares, Atmos. Environ., 2001, 35, 3075.

21 P. P. A. Malley and T. F. Kahan, J. Phys. Chem. A, 2014, 118, 1638.

22 D. F. Ogletree, H. Bluhm, E. D. Hebenstreit and M. Salmeron, Nucl. Instrum. Methods Phys. Res., Sect. A, 2009, 601, 151.

23 H. Bluhm, K. Andersson, T. Araki, K. Benzerara, G. Brown, J. Dynes, S. Ghosal, M. Gilles, H. Hansen, J. Hemminger, A. Hitchcock, G. Ketteler, A. Kilcoyne, E. Kneedler, J. Lawrence, G. Leppard, J. Majzlan, B. Mun, S. Myneni, A. Nilsson, H. Ogasawara, D. Ogletree, K. Pecher, M. Salmeron, D. Shuh, B. Tonner, T. Tyliszczak, T. Warwick and T. Yoon, J. Electron. Spectrosc. Relat. Phenom., 2006, 150, 86.

24 W. Wagner and A. Pruss, J. Phys. Chem. Ref. Data, 2002, 31, 387.

25 J. M. Lafferty, Foundations of Vacuum Science and Technology, Wiley, New York, 1998.

26 J. F. Moulder, W. F. Stickle, P. E. Sobol and K. D. Bomben, Handbook of X-ray Photoelectron Spectroscopy, Physical Electronics, Inc., Eden Prairie, MN, 1995.

27 Y. Joseph, C. Kuhrs, W. Ranke and W. Weiss, Surf. Sci., 1999, 433, 114.

28 S. M. Clegg and J. P. D. Abbatt, J. Phys. Chem. A, 2001, 105, 6630. 29 B. Collignon and S. Picaud, Chem. Phys. Lett., 2004, 393, 457. 30 P. Jedlovszky, L. Partay, P. N. M. Hoang, S. Picaud, P. von Hessberg and J. N. Crowley, J. Am. Chem. Soc., 2006, 128, 15300. 